of copper, or by a yellow precipitate of the hydrated oxide. If the solution of Fehling's solution becomes turbid on being boiled, it is well to discard the sample, for the turbidity is evidence of a chemical decomposition which may vitiate the test. The solution will keep for a great length of time if its ingredients are pure, but, generally speaking, it is wise to keep the copper and the soda solutions in separate bottles until the mixture is required.

Regarding the fallacies attending tests that involve the reduction of copper salts. There are several substances, normal constituents of urine, which are capable of effecting this. Of these the chief are uric acid and urates, kreatinin and glycuronic acid. But, generally speaking, the reducing capacity of these substances is sufficiently small for them to require prolonged boiling before evidence of reduction appears. In the case of sugar, on the other hand, reduction is evident before the boilingpoint is reached; indeed, it will take place in the cold, but only after a lengthened interval. In this earlier appearance of reduction in the case of sugar lies the explanation of the advice that the mixture of urine and Fehling's solution should not be boiled.

Ammoniacal urine should not be tested with Fehling's solution, for if the quantity of sugar present be small, the oniy result will be a slight fading of colour, which is easily overlooked.

It is important not to add too much urine, for in such a case the caustic alkali may lead to the precipitation of earthy phosphates which may simulate a reduction product.

Occasionally one meets with cases in which the specimen, on being cooled, appears as a turbid fluid of a turquoise or greenish-yellow colour. For such a case the other three methods are useful corroboratives.

Nylander's test.-This is described by the author as an excellent test capable of detecting .1 per cent. of sugar. The reagent is made as follows. With the aid of gentle heat a solution is made according to the accompanying prescription.

Bismuth subnitrate

Rochelle salt

Caustic soda

Distilled water

$\begin{array}{lr}\text {.. } & 2 \text { grammes } \\ \text {.. } & 4 \\ \text {.. } 10 \\ \text {.. } 100 \text { c.c. } ",\end{array}$

This forms a colourless liquid which will keep indefinitely. The test is applied by boiling together in a test-tube, for two minutes, three drachms of urine and twenty drops of the reagent. A positive reaction is shown by a blackening of the mixture due to the formation of the sulphide of bis-
muth.

The fermentation test affords complete proof of the presence of sugar. The fallacies that attend its use are a possible inactivity of the yeast employed, and the presence of starch in the yeast, as commonly happens in the commercial product. In consequence of these possibilities the test is inconclusive unless control experiments are simultaneously conducted. The test is rather too complicated for routine work; moreover, it is unsatisfactory when the percentage of sugar present falls below .3 per cent.

The phenylhydrazine test is " the most serviceable and one of the most delicate tests we have." It is carried out as follows: five grains of phenylhydrazine hydrochloride and ten grains of sodium acetate are added to half an ounce of urine; the whole is placed in a water-bath, and boiled for half an hour. On being cooled, the mixture, if it contains sugar, will deposit fans, stars, and sheaves of phenylglucosazone crystals, which are easily identified under a low power of the microscope. This test will detect a quantity of sugar so small as to be by some considered physiological ; but in such a case the mass of crystals is very insignificant.

In addition to the above fallacies it is well to remember that there is a large number of drugs known to affect the action of Fehling's solution. Such, for instance, are alcohol in large quantity, arsenic, aspirin, chloral hydrate and butyl-chloral hydrate, camphor, chloroform, carbolic acid, morphine, salol and salicylic acid, sulphonal, and many coal-tar products. Nylander's solution is also influenced by arsenic, carbolic acid, iodides and mercury, quinine, rhubarb, salicylic acid, sulphur, and turpentine. A positive reaction is also produced by melanin if that be present in the urine.

1 Birmingham.Med. Rev., October 1905.

\section{THE TREATMENT OF FRACTURE OF THE PATELLA.}

A $\mathrm{T}$ a meeting of the Medico-Chirurgical Society of Edinburgh on December 6, Mr. G. G. Hamilton read a paper on the treatment of fractured patella by transverse wiring. He said that, while operations on the knee-joint require the most scrupulous asepsis, if this condition is fulfilled wiring gives the best results in the shortest time and is therefore that which should be chosen for patients in whom the limb will be subjected to severe strain in the course of their occupation. A semi-lunar incision is made with the convexity downwards, which at its lowest part reaches to the lower fragment. The joint is opened and cleared of blood and any loose fragments of bone, but the patella should not be separated from the soft parts. The two fragments are then drilled transversely and brought together by strong silver wire. The patient may be allowed to move the knee about ten days after the operation. Radiographs shown by the lantern, demonstrated that osseous union was readily secured in this way, while by other methods though fairly good functional results were obtained, there was often great separation of the fragments and bony union was extremely rare. A lad was shown who had fractured both patellæ and was treated by operation a year ago with excellent results. In another patient, who had sustained a fracture of the left patella nine years ago and wore a splint for many months, a fracture on the right side had been wired a month ago. This man stated that his right leg was as useful after a month's treatment as the left had been after eighteen months. In a patient who died of apoplexy some weeks after operation the wound had healed and the fragments of the patella were found to be firmly united. 